PROCEEDINGS OF THE

AMERICAN MATHEMATICAL SOCIETY

Volume 133, Number 10, Pages 2977-2984

S 0002-9939(05)07676-8

Article electronically published on May 13, 2005

\title{
A NOTE ON WEYL'S THEOREM
}

\author{
XIAOHONG CAO, MAOZHENG GUO, AND BIN MENG
}

(Communicated by N. Tomczak-Jaegermann)

\begin{abstract}
The Kato spectrum of an operator is deployed to give necessary and sufficient conditions for Browder's theorem to hold.
\end{abstract}

\section{INTRODUCTION}

Weyl's theorem holds, for a bounded linear operator $A$ on a Banach space $X$, provided

$$
\sigma(A) \backslash \sigma_{w}(A)=\pi_{00}(A),
$$

where $\sigma(A)$ is the usual spectrum of $A, \sigma_{w}(A)$ is the Weyl spectrum, collecting complex numbers $\lambda$ for which $A-\lambda I$ fails to be Fredholm of index zero, and $\pi_{00}(A)$ is the isolated points of the spectrum which are eigenvalues of finite multiplicity. About half of this condition has been [5] christened "Browder's theorem holds":

$$
\sigma(A) \subseteq \sigma_{w}(A) \cup \pi_{00}(A) ;
$$

equivalently

$$
\sigma_{b}(A) \subseteq \sigma_{w}(A),
$$

where the Browder spectrum $\sigma_{b}(A)$ collects complex numbers $\lambda$ for which $A-\lambda I$ fails to be Fredholm of finite ascent and descent. Of course these inclusion are automatically equalities. In some sense "orthogonal" to finite ascent and descent is the Saphar condition of "perfection" [10, also known as "hyperexactness" [6]: we might call $A: X \longrightarrow X$ a Saphar operator provided

$$
N(A) \subseteq \bigcap_{n=1}^{\infty} R\left(A^{n}\right), \quad \text { equivalently } \quad \bigcup_{n=1}^{\infty} N\left(A^{n}\right) \subseteq R(A) .
$$

Obviously Saphar operators of finite ascent are one-one, and Saphar operators of finite descent are onto; the Saphar condition picks out those Fredholm operators for which $0 \in \mathbb{C}$ is not a jump point for the index. The Saphar condition also converts the closed range condition into the basis [7, [6] for another kind of spectrum: the Kato spectrum $\sigma_{k}(A)$ collects those complex numbers $\lambda$ for which

$$
A-\lambda I \text { fails to be Saphar with closed range. }
$$

Received by the editors June 3, 2003 and, in revised form, February 4, 2004.

2000 Mathematics Subject Classification. Primary 47A10, 47A53, 47A55.

Key words and phrases. Browder's theorem, a-Browder's theorem, Weyl's theorem, a-Weyl's theorem. 
It is the union of the Weyl and the Kato spectrums which serve to characterize the condition "Browder's theorem holds". We begin with a lemma.

Lemma 1.1. If $A: X \longrightarrow X$ is bounded linear on a Banach space, then

$$
\sigma(A) \subseteq \sigma_{b}(A) \cup \sigma_{k}(A)
$$

and

$$
\text { iso } \sigma(A) \subseteq \sigma_{w}(A) \cup \pi_{00}(A) \text {. }
$$

Also

$$
\text { iso } \sigma_{k}(A) \subseteq \sigma_{w}(A)
$$

and

$$
\partial \sigma(A) \subseteq \sigma_{k}(A) .
$$

Proof. (6) is the observation that Saphar operators of finite ascent and descent are invertible while (7) uses the punctured neighborhood theorem to make the point that the Saphar condition rules out index jumps. (8) is also the punctured neighborhood theorem, while (9) uses gap theory [11, [6].

\section{BROWDER'S THEOREM AND WEYL'S THEOREM}

Let $\Phi_{+}(X)\left(\Phi_{-}(X)\right)$ be the set of all upper (lower) semi-Fredholm operators. If $A \in \Phi_{+}(X)$, then $A-\lambda I$ is an upper semi-Fredholm operator with index $\operatorname{ind}(A-\lambda I)=\operatorname{ind}(A)$ and $N(A-\lambda I) \subseteq \bigcap_{n=1}^{\infty} R\left[(A-\lambda I)^{n}\right]$ if $|\lambda|$ is sufficiently small. In fact, if $A \in \Phi_{+}(X)$, it is well known that $A-\lambda I \in \Phi_{+}(X)$ and $\operatorname{ind}(A-\lambda I)=\operatorname{ind}(A)$ if $|\lambda|$ is sufficiently small, $Y=\bigcap_{n=1}^{\infty} R\left(A^{n}\right)$ is closed and $A Y=Y$. Without loss of generality, suppose that $Y \neq 0$. Let $A_{1}=\left.A\right|_{Y}$; then $A_{1}$ is surjective and hence $A_{1} \in \Phi_{-}(Y)$. By perturbation theory of a semi-Fredholm operator, $A_{1}-\lambda I$ is surjective, that is, $\left(A_{1}-\lambda I\right) Y=Y$ if $|\lambda|$ is sufficiently small. Then $Y=\bigcap_{n=1}^{\infty} R\left[\left(A_{1}-\lambda I\right)^{n}\right] \subseteq \bigcap_{n=1}^{\infty} R\left[(A-\lambda I)^{n}\right]$ if $|\lambda|$ is sufficiently small. Since $N(A-\lambda I) \subseteq Y$, it follows that $N(A-\lambda I) \subseteq \bigcap_{n=1}^{\infty} R\left[(A-\lambda I)^{n}\right]$.

The Weyl and Kato spectrums together delineate the Browder's theorem condition:

Theorem 2.1. Necessary and sufficient for Browder's theorem to hold, for bounded linear $A: X \longrightarrow X$, is the inclusion

$$
\sigma(A) \subseteq \sigma_{w}(A) \cup \sigma_{k}(A) .
$$

Proof. One direction is trivial. With no conditions

$$
\sigma_{w}(A) \cup \pi_{00}(A) \subseteq \sigma_{w}(A) \cup \sigma_{k}(A) .
$$

The left-hand side is a subset of

$$
\sigma_{w}(A) \cup\left(\text { iso } \sigma(A) \backslash \sigma_{w}(A)\right)=\sigma_{w}(A) \cup\left(\sigma(A) \backslash \sigma_{b}(A)\right),
$$

which is a subset of the right-hand side. Conversely if (10) holds, then

$\lambda$ is not in $\sigma_{w}(A) \Longrightarrow \lambda$ is not in acc $\left(\sigma_{w}(A) \cup \sigma_{k}(A)\right)=\operatorname{acc} \sigma(A)$, 
and hence

giving (2).

$$
\sigma(A) \backslash \sigma_{w}(A) \subseteq \text { iso } \sigma(A) \backslash \sigma_{w}(A)=\sigma(A) \backslash \sigma_{b}(A),
$$

In general we cannot reverse the inclusion (11): for example [5. Example 6] the direct sum of the forward and backward shifts is Weyl and not Browder or Saphar.

Let $H(A)$ be the class of all complex-valued functions which are analytic on a neighborhood of $\sigma(A)$ and let $\sigma_{1}(A)=\sigma_{w}(A) \cup \sigma_{k}(A), \rho_{1}(A)=\mathbb{C} \backslash \sigma_{1}(A)$.

Corollary 2.2. If Browder's theorem holds for $A \in B(X)$ and for $B \in B(X)$ and if $f \in H(A)$, then

$$
\text { Browder's theorem holds for } f(A) \Longleftrightarrow \sigma_{1}(f(A))=f\left(\sigma_{1}(A)\right)
$$

and

$$
\text { Browder's theorem holds for } A \oplus B \Longleftrightarrow \sigma_{1}(A) \cup \sigma_{1}(B)=\sigma_{1}(A \oplus B) \text {. }
$$

Proof. Using Theorem 4 in [5] and the Kato spectrum mapping theorem, we get that for any $f \in H(A)$,

Browder's theorem holds for $f(A) \Longrightarrow \sigma_{1}(f(A))=\sigma_{w}(f(A)) \cup \sigma_{k}(f(A))$

$$
=f\left(\sigma_{w}(A)\right) \cup f\left(\sigma_{k}(A)\right)=f\left(\sigma_{1}(A)\right)
$$

and

Browder's theorem holds for $A \oplus B \Longrightarrow \sigma_{1}(A \oplus B)=\sigma_{w}(A \oplus B) \cup \sigma_{k}(A \oplus B)$

$$
\begin{aligned}
& =\sigma_{w}(A) \cup \sigma_{w}(B) \cup \sigma_{k}(A) \cup \sigma_{k}(B) \\
& =\sigma_{1}(A) \cup \sigma_{1}(B) .
\end{aligned}
$$

Conversely, for every $f \in H(A)$, if $\sigma_{1}(f(A))=f\left(\sigma_{1}(A)\right)$, then $\sigma_{1}(f(A))=f\left(\sigma_{1}(A)\right)$ $=f(\sigma(A))=\sigma(f(A))$, which means that Browder's theorem holds for $f(A)$. And if $\sigma_{1}(A \oplus B)=\sigma_{1}(A) \cup \sigma_{1}(B)$, then $\sigma_{1}(A \oplus B)=\sigma(A) \cup \sigma(B)=\sigma(A \oplus B)$. Therefore Browder's theorem holds for $A \oplus B$.

Also we can proof the following result:

Corollary 2.3. The following statements are equivalent:

(a) Browder's theorem holds for A;

(b) $\sigma(A) \backslash \sigma_{w}(A) \subseteq \sigma_{1}(A)$;

(c) $\sigma(A) \backslash \sigma_{w}(A) \subseteq$ iso $\sigma_{1}(A)$;

(d) $\sigma_{b}(A) \subseteq \sigma_{1}(A)$;

(e) $\sigma(A) \backslash \sigma_{w}(A) \subseteq \sigma_{k}(A)$;

(f) $\sigma(A) \backslash \sigma_{k}(A) \subseteq$ iso $\sigma_{k}(A)$.

Corollary 2.4. Weyl's theorem holds for $A$ if and only if

$$
\text { iso } \sigma_{1}(A) \cap \pi_{0 f}(A)=\sigma(A) \backslash \sigma_{w}(A),
$$

where $\pi_{0 f}(A)$ denotes the set of eigenvalues of $A$ of finite multiplicity.

Proof. $\Longrightarrow$. Weyl's theorem implies Browder's theorem; then $\sigma(A)=\sigma_{1}(A)$. Thus iso $\sigma_{1}(A) \cap \pi_{0 f}(A)=$ iso $\sigma(A) \cap \pi_{0 f}(A)=\pi_{00}(A)=\sigma(A) \backslash \sigma_{w}(A)$.

$\Longleftarrow$. Clearly, $\pi_{00}(A) \subseteq$ iso $\sigma_{1}(A) \cap \pi_{0 f}(A)=\sigma(A) \backslash \sigma_{w}(A)$. Conversely, if $\lambda_{0} \in$ $\sigma(A) \backslash \sigma_{w}(A)$, then there exists $\epsilon>0$ such that $\lambda \in \rho_{1}(A)$ if $0<\left|\lambda-\lambda_{0}\right|<\epsilon$. We claim that $\lambda \in \mathbb{C} \backslash \sigma(A)$ if $0<\left|\lambda-\lambda_{0}\right|<\epsilon$. In fact, if there exists $\lambda_{1}$ such that 
$0<\left|\lambda_{1}-\lambda_{0}\right|<\epsilon$ and $\lambda_{1} \in \sigma(A)$, then $\lambda_{1} \in \sigma(A) \backslash \sigma_{w}(A)=$ iso $\sigma_{1}(A) \cap \pi_{0 f}(A)$. Then $\lambda_{1} \in \sigma_{1}(A)$, a contradiction. It follows that $\lambda_{0} \in$ iso $\sigma(A)$ and hence $\lambda_{0} \in \pi_{00}(A)$.

From the above proof, we get that $\sigma(A) \backslash \sigma_{w}(A)=\pi_{00}(A)$. Then Weyl's theorem holds for $A$.

In general the spectral mapping theorem is liable to fail for the spectrum $\sigma_{1}(\cdot)$, but there is only inclusion:

Lemma 2.5. For any $f \in H(A), \sigma_{1}(f(A)) \subseteq f\left(\sigma_{1}(A)\right)$.

Proof. For any $f \in H(A)$, since $\sigma_{w}(f(A)) \subseteq f\left(\sigma_{w}(A)\right)$ and $\sigma_{k}(f(A))=f\left(\sigma_{k}(A)\right)$ [11, Satz 6], it follows that

$$
\begin{aligned}
\sigma_{1}(f(A))=\sigma_{w}(f(A)) \cup \sigma_{k}(f(A)) & \subseteq f\left(\sigma_{w}(A)\right) \cup f\left(\sigma_{k}(A)\right) \\
& =f\left(\sigma_{w}(A) \cup \sigma_{k}(A)\right)=f\left(\sigma_{1}(A)\right) .
\end{aligned}
$$

We use $\sigma_{e}(A)$ to denote the essential spectrum of operator $A$.

Theorem 2.6. $\operatorname{ind}(A-\lambda I) \cdot \operatorname{ind}(A-\mu I) \geq 0$ for each pair $\lambda, \mu \in \mathbb{C} \backslash \sigma_{e}(A)$ if and only if

$$
\sigma_{1}(f(A))=f\left(\sigma_{1}(A)\right) \text { for each } f \in H(A) .
$$

Proof. Suppose that $i n d(A-\lambda I) \cdot \operatorname{ind}(A-\mu I) \geq 0$ for each pair $\lambda, \mu \in \mathbb{C} \backslash \sigma_{e}(A)$. From Theorem 5 in [5], we know that the spectral mapping theorem holds for the Weyl spectrum. Then

$$
\begin{aligned}
\sigma_{1}(f(A)) & =\sigma_{w}(f(A)) \cup \sigma_{k}(f(A))=f\left(\sigma_{w}(A)\right) \cup f\left(\sigma_{k}(A)\right) \\
& =f\left(\sigma_{w}(A) \cup \sigma_{k}(A)\right)=f\left(\sigma_{1}(A)\right) .
\end{aligned}
$$

Conversely, if there exists $\lambda_{0}, \mu_{0} \in \mathbb{C} \backslash \sigma_{e}(A)$ for which

$$
\operatorname{ind}\left(A-\lambda_{0} I\right)=-m<0<k=\operatorname{ind}\left(A-\mu_{0} I\right) .
$$

Let

$$
p(\lambda)=\left(\lambda-\lambda_{0}\right)^{k}\left(\lambda-\mu_{0}\right)^{m} .
$$

Then $p(A)=\left(A-\lambda_{0} I\right)^{k}\left(A-\mu_{0} I\right)^{m}$ is a Weyl operator. By the punctured neighborhood theorem, there exists $\delta>0$ such that $h \in \rho_{1}(p(A))$ if $0<|h|<\delta$. Similarly, there exists $\epsilon>0$ such that $0<|p(\lambda)|<\delta, 0<|p(\mu)|<\delta, A-\lambda I$ is Fredholm with index $-m$ and $A-\mu I$ is Fredholm operator with index $k$ if $0<\left|\lambda-\lambda_{0}\right|<\epsilon$ and $0<\left|\mu-\mu_{0}\right|<\epsilon$. Then $\lambda \in \sigma_{1}(A)$ and $\mu \in \sigma_{1}(A)$ if $0<\left|\lambda-\lambda_{0}\right|<\epsilon$ and $0<\left|\mu-\mu_{0}\right|<\epsilon$. Thus $p(\lambda) \in p\left(\sigma_{1}(A)\right)=\sigma_{1}(p(A))$ and $p(\mu) \in p\left(\sigma_{1}(A)\right)=\sigma_{1}(p(A))$ if $0<\left|\lambda-\lambda_{0}\right|<\epsilon$ and $0<\left|\mu-\mu_{0}\right|<\epsilon$. It is in contradiction to the fact that $p(\lambda) \in \rho_{1}(p(A))$ and $p(\mu) \in \rho_{1}(p(A))$.

Then we have that:

Corollary 2.7. If Browder's theorem holds for A, then

$$
\operatorname{ind}(A-\lambda I) \cdot \operatorname{ind}(A-\mu I) \geq 0 \text { for each pair } \lambda, \mu \in \mathbb{C} \backslash \sigma_{e}(A)
$$

if and only if Browder's theorem holds for $f(A)$ for every $f \in H(A)$.

Corollary 2.8. $\sigma_{1}(f(A))=f\left(\sigma_{1}(A)\right)$ for every $f \in H(A)$ if and only if $\sigma_{w}(f(A))=$ $f\left(\sigma_{w}(A)\right)$ for every $f \in H(A)$.

Proof. Using Theorem 2.6 above and Theorem 5 in [5], we get the result. 


\section{A-BROWDER'S THEOREM AND A-WEYL'S THEOREM}

In this section, we go on to make an analogous discussion of "a-Browder's theorem".

Let $\Phi_{+}^{-}(X)$ be the class of all $A \in \Phi_{+}(X)$ with $\operatorname{ind}(A) \leq 0$, and for any $A \in$ $B(X)$, let

$$
\sigma_{e a}(A)=\left\{\lambda \in \mathbb{C}: A-\lambda I \text { is not in } \Phi_{+}^{-}(X)\right\}
$$

and

$\sigma_{a b}(A)=\{\lambda \in \mathbb{C}: A-\lambda I$ is not upper semi-Fredholm with finite ascent $\}$.

We call $\sigma_{e a}(A)$ the essential approximate point spectrum of $A$ and $\sigma_{a b}(A)$ the Browder essential approximate point spectrum of $A$.

Similar to Weyl's theorem and Browder's theorem, there is a-Weyl's theorem and a-Browder's theorem [1], 8]. We say that a-Weyl's theorem holds for $A$ if there is equality

$$
\sigma_{a}(A) \backslash \sigma_{e a}(A)=\pi_{00}^{a}(A),
$$

where $\sigma_{a}(A)$ is the approximate point spectrum and $\pi_{0}^{a}(A)$ the isolated points of $\sigma_{a}(A)$ which are eigenvalues of finite multiplicity, and that a-Browder's theorem holds for $A$ if there is equality

$$
\sigma_{e a}(A)=\sigma_{a b}(A) .
$$

It is known [1, 2] that if $A \in B(X)$, then we have

$$
\text { a-Weyl's theorem } \Longrightarrow \text { Weyl's theorem } \Longrightarrow \text { Browder's theorem }
$$

and

a-Weyl's theorem $\Longrightarrow$ a-Browder's theorem $\Longrightarrow$ Browder's theorem.

Let $\sigma_{2}(A)=\sigma_{e a}(A) \cup \sigma_{k}(A)$ and let $\rho_{2}(A)=\mathbb{C} \backslash \sigma_{2}(A)$. We can prove that

$$
\sigma_{a}(A)=\sigma_{2}(A) \cup \sigma_{a b}(A)=\sigma_{k}(A) \cup \sigma_{a b}(A) .
$$

Theorem 3.1. Necessary and sufficient for a-Browder's theorem to hold, for bounded linear $A: X \longrightarrow X$, is the inclusion

$$
\sigma_{a}(A) \subseteq \sigma_{e a}(A) \cup \sigma_{k}(A) .
$$

Proof. Similar to the proof of Theorem 2.1, one direction is trivial. With no conditions

$$
\sigma_{e a}(A) \cup \pi_{00}^{a}(A) \subseteq \sigma_{e a}(A) \cup \sigma_{k}(A) ;
$$

the left-hand side is a subset of

$$
\sigma_{e a}(A) \cup\left(\text { iso } \sigma_{a}(A) \backslash \sigma_{e a}(A)\right)=\sigma_{e a}(A) \cup\left(\sigma_{a}(A) \backslash \sigma_{a b}(A)\right),
$$

which is a subset of the right-hand side. Conversely, if $\sigma_{a}(A) \subseteq \sigma_{e a}(A) \cup \sigma_{k}(A)$, then

$$
\lambda \text { is not in } \sigma_{e a}(A) \Longrightarrow \lambda \text { is not in acc }\left(\sigma_{e a}(A) \cup \sigma_{k}(A)\right)=\operatorname{acc} \sigma_{a}(A),
$$

and hence

$$
\sigma_{a}(A) \backslash \sigma_{e a}(A) \subseteq \text { iso } \sigma_{a}(A) \backslash \sigma_{e a}(A)=\sigma_{a}(A) \backslash \sigma_{a b}(A),
$$

which means that $\sigma_{e a}(A)=\sigma_{a b}(A)$. Then a-Browder's theorem holds for $A$. 
For approximate point spectrum, there is spectral mapping theorem, that is, for every $f \in H(A), \sigma_{a}(f(A))=f\left(\sigma_{a}(A)\right)$. Then:

Corollary 3.2. If a-Browder's theorem holds for $A \in B(X)$ and $B \in B(X)$ and if $f \in H(A)$, then

$$
\text { a-Browder's theorem holds for } f(A) \Longleftrightarrow \sigma_{2}(f(A))=f\left(\sigma_{2}(A)\right)
$$

and

a-Browder's theorem holds for $A \oplus B \Longleftrightarrow \sigma_{2}(A) \cup \sigma_{2}(B)=\sigma_{2}(A \oplus B)$.

Proof. Using Theorem 3.1, for every $f \in H(A)$, a-Browder's theorem holds for $f(A) \Longleftrightarrow \sigma_{2}(f(A))=\sigma_{a}(f(A))=f\left(\sigma_{a}(A)\right)=f\left(\sigma_{2}(A)\right)$. Also,

a-Browder's theorem holds for $A \oplus B \quad \Longrightarrow \quad \sigma_{2}(A \oplus B)=\sigma_{a}(A \oplus B)$

$$
=\sigma_{a}(A) \cup \sigma_{a}(B)=\sigma_{2}(A) \cup \sigma_{2}(B) \text {. }
$$

Similarly, we can prove that:

Corollary 3.3. The following statements are equivalent:

(a) a-Browder's theorem holds for A;

(b) $\sigma_{a}(A) \backslash \sigma_{e a}(A) \subseteq \sigma_{2}(A)$;

(c) $\sigma_{a}(A) \backslash \sigma_{e a}(A) \subseteq$ iso $\sigma_{2}(A)$;

(d) $\sigma_{a b}(A) \subseteq \sigma_{2}(A)$;

(e) $\sigma_{a}(A) \backslash \sigma_{e a}(A) \subseteq \sigma_{k}(A)$;

(f) $\quad \sigma_{a}(A) \backslash \sigma_{e a}(A) \subseteq$ iso $\sigma_{k}(A)$.

Corollary 3.4. a-Weyl's theorem holds for $A$ if and only if

$$
\text { iso } \sigma_{2}(A) \cap \pi_{0 f}(A)=\sigma_{a}(A) \backslash \sigma_{e a}(A) \text {. }
$$

We know that the inclusion $\sigma_{e a}(f(A)) \subseteq f\left(\sigma_{e a}(A)\right)$ holds $[9$; then we can prove that:

Lemma 3.5. For any $f \in H(A), \sigma_{2}(f(A)) \subseteq f\left(\sigma_{2}(A)\right)$.

Theorem 3.6. $\operatorname{ind}(A-\lambda I) \operatorname{ind}(A-\mu I) \geq 0$ for each pair $\lambda, \mu \in \mathbb{C}$ such that $A-\lambda I \in \Phi_{+}(X)$ and $A-\mu I \in \Phi_{+}(X)$ if and only if

$$
\sigma_{2}(f(A))=f\left(\sigma_{2}(A)\right) \text { for every } f \in H(A) .
$$

Proof. Suppose that $\operatorname{ind}(A-\lambda I) \operatorname{ind}(A-\mu I) \geq 0$ for each pair $\lambda, \mu \in \mathbb{C}$ such that $A-\lambda I \in \Phi_{+}(X)$ and $A-\mu I \in \Phi_{+}(X)$. We only need to prove that $f\left(\sigma_{2}(A)\right) \subseteq$ $\sigma_{2}(f(A))$ for any $f \in H(A)$.

Let $\mu \in f\left(\sigma_{2}(A)\right)$ and suppose that $\mu$ is not in $\sigma_{2}(f(A))$. Since $\sigma_{k}(f(A)) \subseteq$ $\sigma_{2}(f(A))$, it follows that $\mu$ is not in $\sigma_{k}(f(A))=f\left(\sigma_{k}(A)\right)$. Define the function $g$ by $g(\lambda)=f(\lambda)-\mu$. Then $g(\lambda) \neq 0$ for all $\lambda \in \sigma_{k}(A)$. Clearly $g(\lambda)$ has zeros in $\sigma(A)$. [11, Satz 3] asserts now that $g$ has only a finite number of zeros in $\sigma(A)$. Let $\lambda_{1}, \lambda_{2}, \cdots, \lambda_{k}$ be these zeros $\left(\lambda_{i} \neq \lambda_{j}\right.$ for $\left.i \neq j\right)$ and $n_{1}, n_{2}, \cdots, n_{k}$ be their respective orders. Suppose

$$
g(\lambda)=f(\lambda)-\mu=\left(\lambda-\lambda_{1}\right)^{n_{1}}\left(\lambda-\lambda_{2}\right)^{n_{2}} \cdots\left(\lambda-\lambda_{k}\right)^{n_{k}} h(\lambda) ;
$$

$h(\lambda)$ has no zeros in $\sigma(A)$, thus $h(A)$ is invertible.

$$
f(A)-\mu I=\left(A-\lambda_{1} I\right)^{n_{1}}\left(A-\lambda_{2} I\right)^{n_{2}} \cdots\left(A-\lambda_{k} I\right)^{n_{k}} h(A) .
$$


Since $f(A)-\mu I \in \Phi_{+}^{-}(X)$, it follows that $A-\lambda_{i} I$ is upper semi-Fredholm and $\sum_{i=1}^{k} \operatorname{ind}\left(A-\lambda_{i} I\right)^{n_{i}} \leq 0$. Then $\operatorname{ind}\left(A-\lambda_{i} I\right) \leq 0$, which means that $A-\lambda_{i} I \in$ $\Phi_{+}^{-}(X)$ for all $i=1,2, \cdots, k$. Since $\lambda_{i} \in \mathbb{C} \backslash \sigma_{k}(A)$, we know that $N\left(A-\lambda_{i} I\right) \subseteq$ $\bigcap_{n=1}^{\infty} R\left[\left(A-\lambda_{i} I\right)^{n}\right]$. Then $\lambda_{i} \in \mathbb{C} \backslash \sigma_{2}(A)$, and therefore $\mu$ is not in $f\left(\sigma_{2}(A)\right)$. It is a contradiction.

Conversely, if there exists $\lambda_{0}, \mu_{0} \in \mathbb{C}$ such that $A-\lambda_{0} I \in \Phi_{+}(X)$ and $A-\mu_{0} I \in$ $\Phi_{+}(X)$ but

$$
\operatorname{ind}\left(A-\lambda_{0} I\right)=-m<0, \quad \operatorname{ind}\left(A-\mu_{0} I\right)=k>0 .
$$

Clearly, $A-\mu_{0} I$ is a Fredholm operator. If $m$ is infinite, let

$$
p(\lambda)=\left(\lambda-\lambda_{0}\right)\left(\lambda-\mu_{0}\right) .
$$

If not, let

$$
p(\lambda)=\left(\lambda-\lambda_{0}\right)^{k}\left(\lambda-\mu_{0}\right)^{m}
$$

Then $p(A) \in \Phi_{+}^{-}(X)$. Thus there exists $\delta>0$ such that $h \in \rho_{2}(p(A))$ if $0<|h|<\delta$. There also exists $\epsilon>0$ such that $A-\mu I$ is a Fredholm operator with index $k>0$ and $0<|p(\mu)|<\delta$ if $0<\left|\mu-\mu_{0}\right|<\epsilon$. Then $p(\mu) \in \rho_{2}(p(A))$. But since $\mu \in \sigma_{2}(A)$, it follows that $p(\mu) \in p\left(\sigma_{2}(A)\right)=\sigma_{2}(p(A))$ if $0<\left|\mu-\mu_{0}\right|<\epsilon$. This is a contradiction.

Then using Theorem 3.6 and Theorem 2.2 in [4, we have that:

Corollary 3.7. $\sigma_{2}(f(A))=f\left(\sigma_{2}(A)\right)$ for each $f \in H(A)$ if and only if

$$
\sigma_{e a}(p(A))=p\left(\sigma_{e a}(A)\right) \text { for each polynomial } p \text {. }
$$

Corollary 3.8. Suppose that a-Browder's theorem holds for A. Then

a-Browder's theorem holds for $f(A)$ for each $f \in H(A)$ if and only if

$$
\sigma_{\text {ea }}(p(A))=p\left(\sigma_{\text {ea }}(A)\right) \text { for each polynomial } p \text {. }
$$

\section{ACKNowledgement}

We are grateful to the referees for helpful comments concerning this paper.

\section{REFERENCES}

1. S.V.Djordjević and D.S.Djordjević, Weyl's theorems: continuity of the spectrum and quasihyponormal operators, Acta Sci. Math. (Szeged) 64 (1998), 259-269. MR.1632013 (2000c:47009)

2. S.V.Djordjević and Y.M.Han, Browder's theorems and spectral continuity, Glasgow Math. J. 42 (2000), 479-486. MR:1793814 (2001h:47003)

3. J.K.Finch, The single valued extension property on a Banach space, Pacific. J. Math. 58 (1975), 61-69. MR0374985 (51:11181)

4. Young Min Han and S.V.Djordjević, A Note on a-Weyl's theorem, J. Math. Anal. Appl. 260 (2001), 200-213. MR1843976 (2002e:47024)

5. R.Harte and Woo Young Lee, Another note on Weyl's theorem, Trans. Amer. Math. Soc. 349 (1997), 2115-2124. MR1407492 (98j:47024)

6. R.E.Harte, On Kato the non-singularity, Studia Math. 117 (1996), 107-114. MR 1367438 (97a:47016)

7. V.Müller, On the regular spectrum, J. Op. Th. 31 (1995), 366-380. MR1331783 (96f:47005)

8. V.Rakočevič, Operators obeying a-Weyl's theorem, Rev. Roumaine Math. Pures Appl. 34 (1989), No. 10, 915-919. MR.1030982 (90k:47009)

9. V.Rakočevic̀, On the essential approximate point spectrum II, Mat. Vesnik 36 (1984), 89-97. MR0880647 (88h:47019) 
10. P.Saphar, Contribution à l'étude des applications linéaires dans un espace de Banach, Bull. Soc. Math. France 92 (1964), 363-384. MR0187095 (32:4549)

11. C.Schmoeger, Ein Spektralabbildungssatz, Arch Math. 55 (1990), 484-489. MR1079997 (92h:47007)

College of Mathematics and Information Science, Shaandi Normal University, Xi'an, 710062, People's Republic of China

E-mail address: xiaohongcao@snnu.edu.cn

Department of Mathematics, Peking University, Beijing, 100871, People's Republic OF CHINA

LMam, School of Mathematical Sciences, Peking University, Beijing, 100871, PeoPLE'S REPUBlic OF ChinA 